\title{
Dynamic Cluster Head Rotation for Data Aggregation in Wireless Sensor Networks
}

\author{
Kiran Kumar ${ }^{1}$, Suma .M ${ }^{2}$ \\ ${ }^{1}$ B M. Tech Student
}

${ }^{2}$ Associate Professor, Department of Electronics and Communication Adichunchanagiri Institute of Technology, Chikmagalur

\begin{abstract}
Routing algorithms are driving the growth of the data transmission in wireless sensor network. Many algorithms proposed for efficient data transferring. This paper uses the scenario and node distribution across the battle field in India. This paper uses clustering algorithms to send the data over different geographic region. During the battle, data gathering and data aggregation to base station is important and critical task. Based on event, clustering algorithm used. This paper assumes that sensor node uniformly distributed and coordinates of the base station and nodes are known. This paper is essential to enable the cluster head based selection scheme used in battle field and the performance of proposed protocol compute intensive and can significantly benefit over the other scheme. Proposed scheme having better data gathering, throughput, packet delivery ratio, and energy, control over head and lifetime than the AODV scheme. The proposed scheme is implemented and stimulated with AODV in NS2.33 simulation shows protocol performance gains in the better over the AODV for lifetime of network and guaranteed data transmission.
\end{abstract}

Keywords: wireless sensor network, clustering, SSPIN, Data gathering, Routing protocol

\section{Introduction}

A wireless sensor network [1][2][4] introduces with a large number of nodes with redundancy existing in; data sensing from environment, data gathering from the nodes, aggregation of processed data etc. In WSN each node is guarantees delivery of specific information to a destination node after specific and typical process along with nodes, more over these nodes can easily transmit the data packet in networks. If it may have enough battery power during the process of data delivery from the source to destination but during the transmission, the large amount of energy is required. Moreover power consumption by electronic devices such as sensor node. Decreasing of energy many tend to exhaust leading to dead node. To overcome this problem, there is a significant concern at the point of individual devices and there have been attempts for data routed in a manner such that energy expenditure during transmission among the node is less to reserve their energy, instead of routing the data to a path that maximizes energy expenditure.

Cluster S-SPIN (cluster version of S-SPIN protocol) scheme is proposed. This scheme guarantees the data delivery from source node to base station. For transferring the data, this scheme does not send the data packet throughout the network but clustering algorithms used for same.

This proposed scheme, executed less number of packet transmission moreover these algorithms will consume less energy and significantly benefited amount of total energy can be saved. Cluster S-SPIN protocol is implemented along with TCL/C++ programming language and NS2.33 simulator.

The rest of the paper has been attempts to organize as follows. In section II, many of the related works attempts have been described with goal of SPIN-S along with its family. Section III describes the problem statement and proposed solution. Section IV describes simulation results of performance gains that have been obtained on performance analysis of Cluster SPIN-S protocol with AODV protocol. Finally, in section V.

\section{Related Work}

Secure SPIN protocol (SPIN-S) has been introduces in three stages like ADV stag, REQ stage and DATA stage.ADV stag enables to each node to checks the advertised data and attempts to find out whether it has already received to a nodes or not. And waits for certain amount of predefined time. If not then node attempts to sends a REQ message in a broadcast manner to all nodes exits in networks. The address of the nodes is specifying in the original advertiser along with header of the message. When a REQ message received to nodes, only the original advertiser nodes will respond and send the original data to the request enabled nodes. Then data aggregation has been done in negotiation mechanism and attempts to ensure the elimination of redundant data. Here a significant concern at the point of individual nodes that it does not establish any path for data transmission from source to base station/sink. Therefore data delivery is not guaranteed in SPIN-S but only some kind of limited security enables. In this phase if cluster head scheme is used then not only a path is established also in guaranteed way data can be transferred to base station.

\section{Problem Statement and Problem Solution}

\section{A. Problem Statement}

Secure SPIN protocol (SPIN-S) has been introduces in three stages like ADV stag, REQ stage and DATA stage.ADV stag enables to each node to checks the advertised data and attempts to find out whether it has already received to a nodes or not. And waits for certain amount of predefined time. If not then node attempts to sends a REQ message in a broadcast manner to all nodes exits in networks. The address of the nodes is specifying in the original advertiser along 
with header of the message. When a REQ message received to nodes, only the original advertiser nodes will respond and send the original data to the request enabled nodes. Then data aggregation has been done in negotiation mechanism and attempts to ensure the elimination of redundant data. Here a significant concern at the point of individual nodes that it does not establish any path for data transmission from source to base station/sink. Therefore data delivery is not guaranteed in SPIN-S but only some kind of limited security enables. In this phase if cluster head scheme is used then not only a path is established also in guaranteed way data can be transferred to base station. Therefore attempts could be made to control number of data transmission along with less energy consumption. Fact is that energy consumption has a greatest portion when enables the nodes to data transmitting or receiving to or from its neighbour nodes. This scheme attempts to provide the guarantee the base station about the reception of data to and from. So new scheme is required to manage the nodes get destroyed earlier in network due to several times used and have ability to gives guarantee of data transmission to base station with increasing the life time of network. All sensor nodes are homogeneous, stationary once the deployed. In the field, location aware. Single base station located in the field. Network model for proposed scheme assumes energy required for running the transmitter and receiver circuitry Eelec as 50nJ/bit.

\section{B. Proposed Solution}

The work of this introduces an efficient cluster head selection scheme for SSPIN Protocol. Aim of the proposed scheme is to reduce the intercommunications distance between the nodes and also distances between the cluster head by means of route shorten method. Proposed scheme divided into the three phase. First phases, cluster head generation among the sensor node within $50 \mathrm{~m}$ distance to find cluster head of each sensor node in the network. To generate the cluster head send the hello packet to all the nodes and select the cluster head within the $50 \mathrm{~m}$ distance. Other phases are negotiation and data transmission. In proposed scheme the role of negotiation phase is to decide the cluster head role rotation after every round of commutation. In last phase, data transferring from cluster to cluster and finally data received to base station. Cluster head consumes more energy as compared to other sensor node. So for increasing the life time of network and sensor node, role of cluster head is rotated among the all sensor nodes in the cluster and cluster head rotation is carried out on the basis of total energy of cluster head and sensor node in a cluster. After every round of data transmission, cluster is decided based on energy.

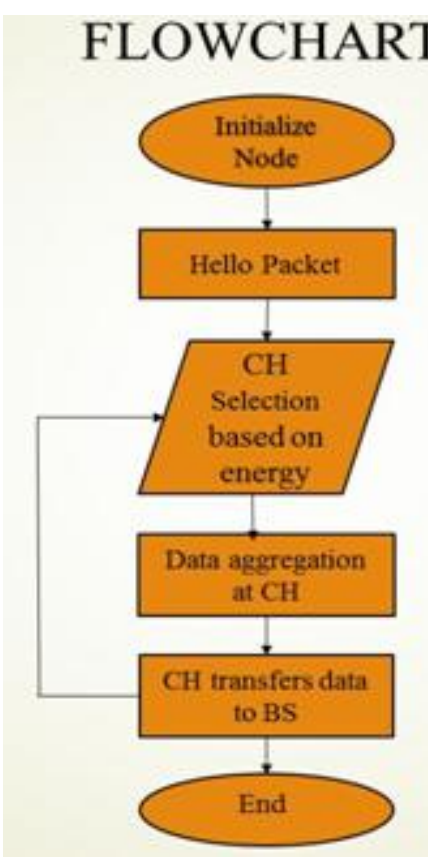

Figure 1: Proposed Cluster SPIN-S Scheme

\section{Simulation Results}

\section{A. Simulation Model}

To evaluate the performance analysis of proposed and AODV by using network simulator NS-2. This scheme guarantees the data delivery from source node to base station. For transferring the data this scheme does not send the data packet throughout the network but clustering algorithms used. The lifetime of the network will be extend then energy used in efficiently. Written in TCL and C++ programming language along with comparison of existing system and proposed scheme.

\section{B. Performance Metrics}

The evaluation is done using the following metrics:

a) Network life time and enabled alive nodes: This metrics able to find out the network life time and number of nodes alive when it takes to travel from source nodes to the base station/sink.

b) Throughput \& packet delivery ratio: This metrics find out the how many control packets produced by each sensor nodes.

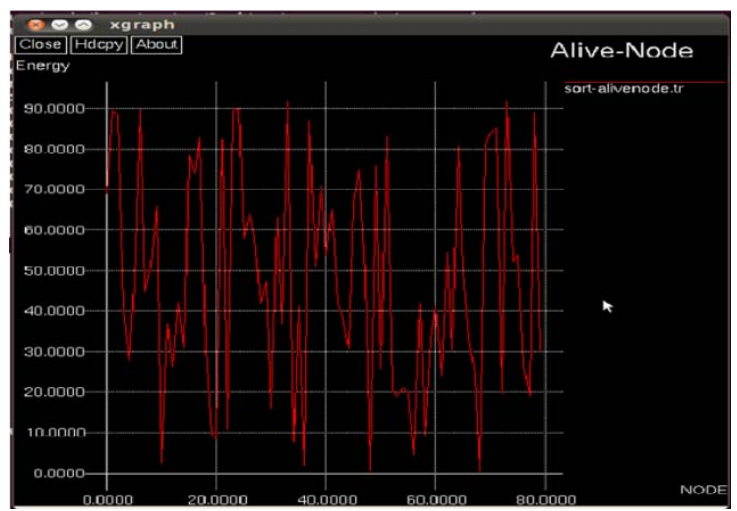

Figure 2: Alive nodes of proposed system. 
International Journal of Science and Research (IJSR)

ISSN (Online): 2319-7064

Index Copernicus Value (2013): 6.14 | Impact Factor (2015): 6.391
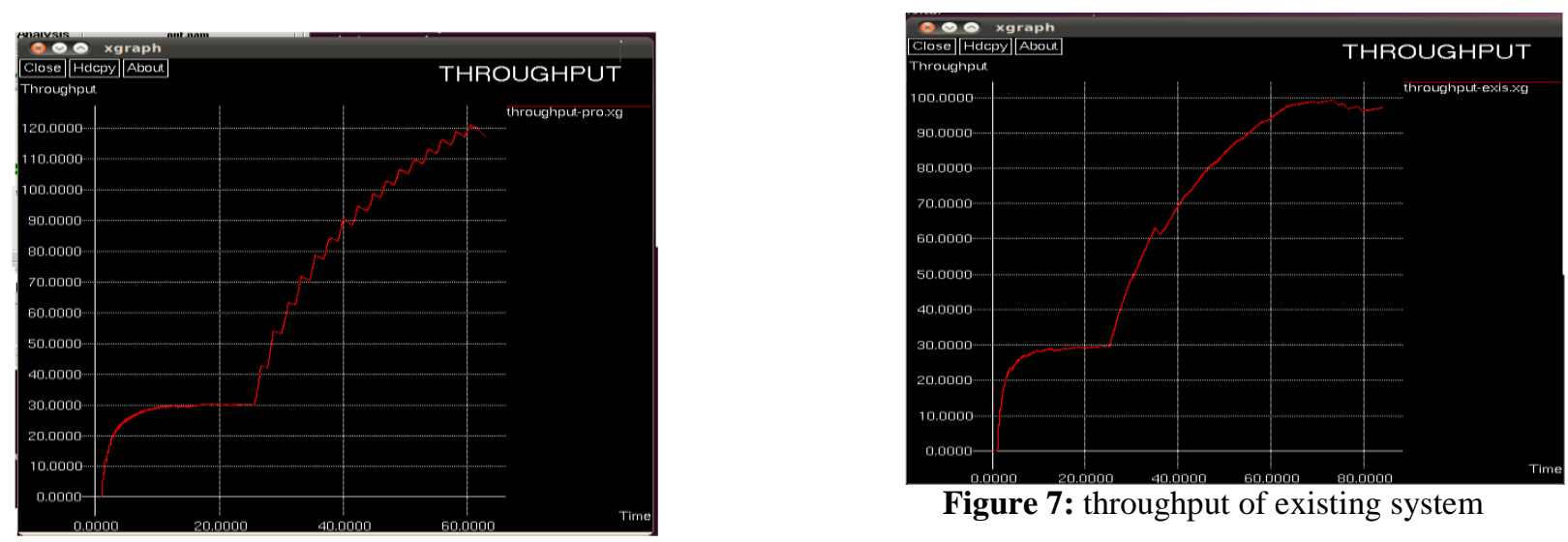

Figure 7: throughput of existing system

Figure 3: throughput of proposed system
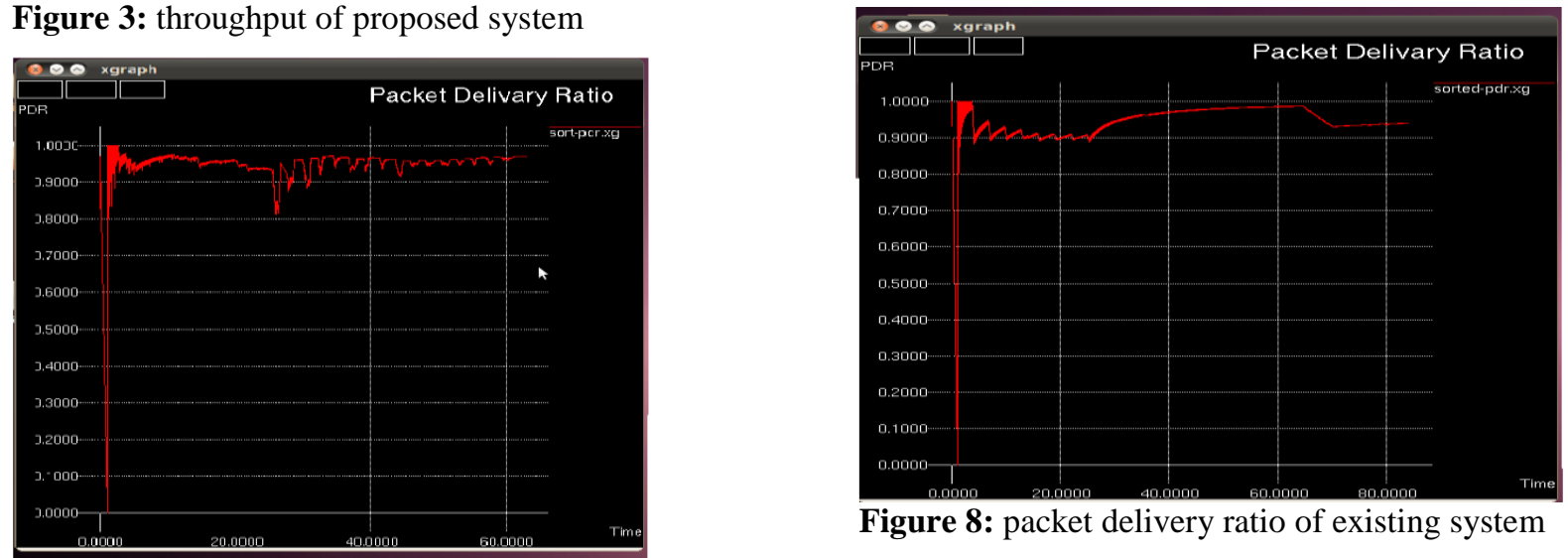

Figure 8: packet delivery ratio of existing system

Figure 4: PDR of proposed system
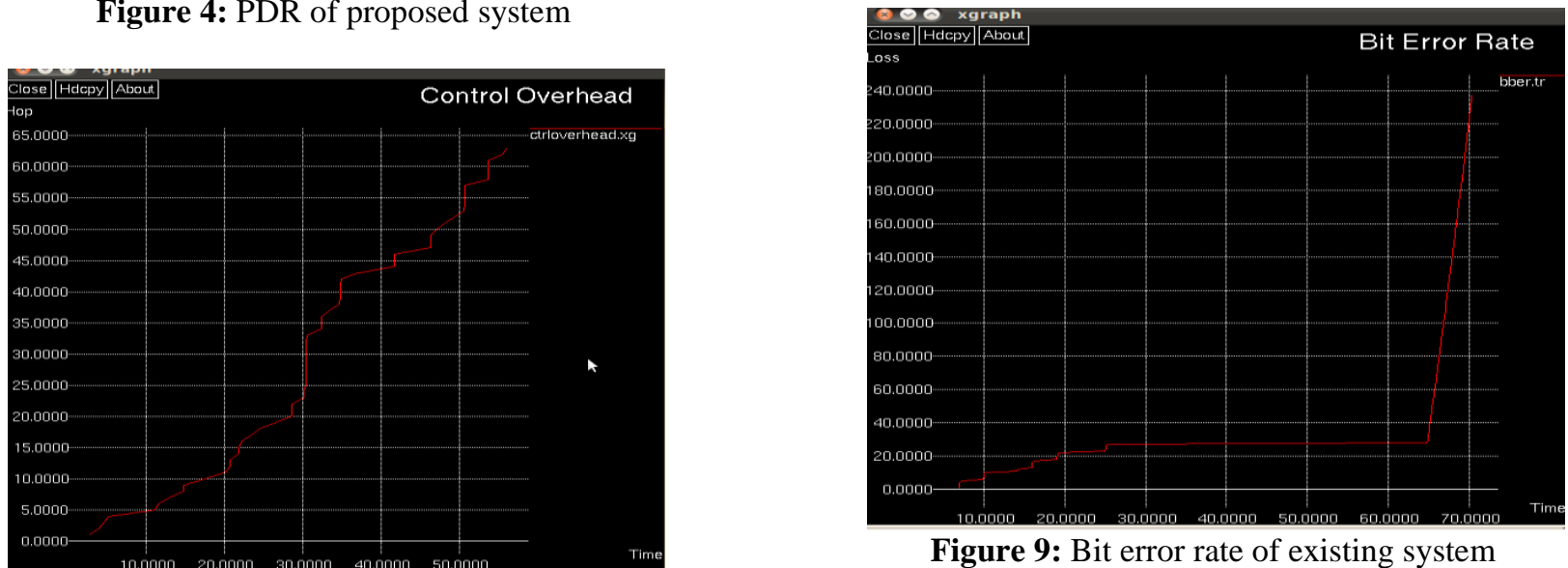

Figure 5: control over head of proposed system

Figure 9: Bit error rate of existing system

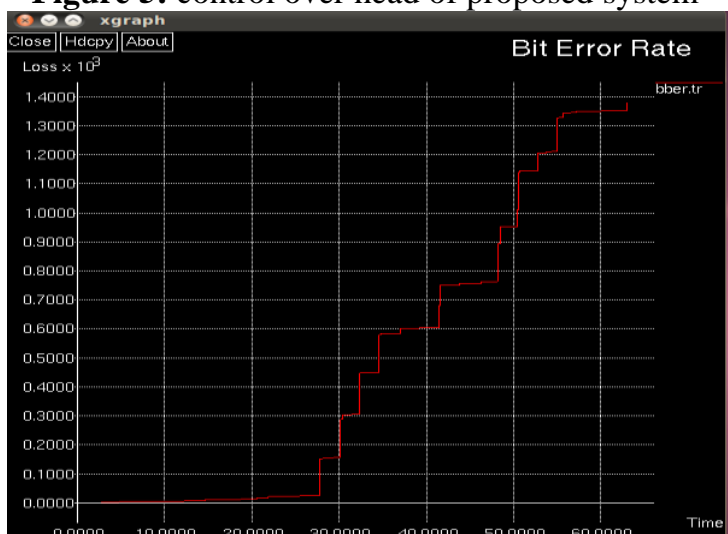

Figure 6: Bit error rate of proposed system

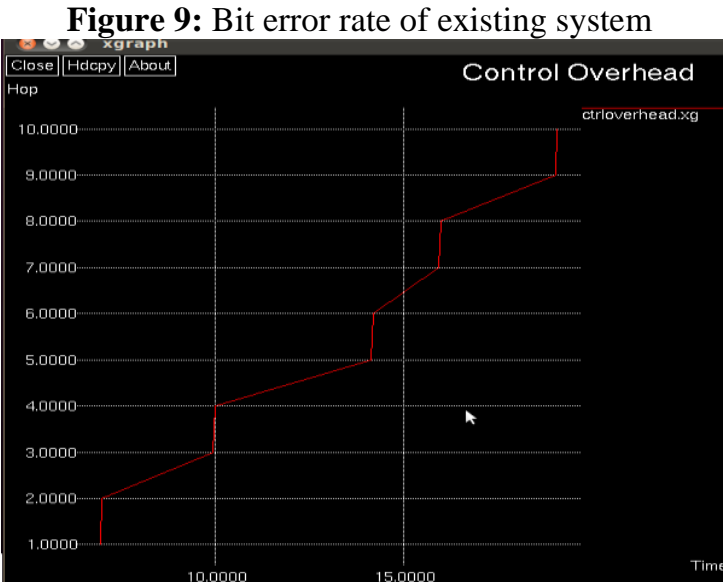

Figure 10: control overhead of existing system 
International Journal of Science and Research (IJSR)

ISSN (Online): 2319-7064

Index Copernicus Value (2013): 6.14 | Impact Factor (2015): 6.391

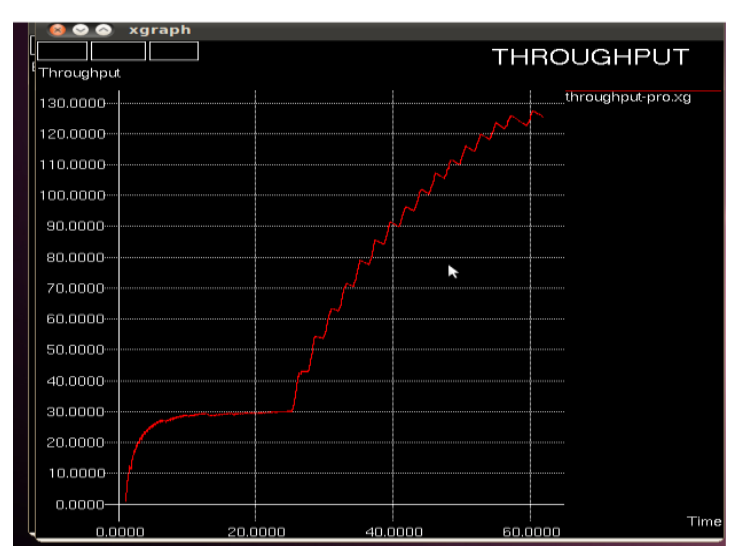

Figure 11: throughput of enhancement system

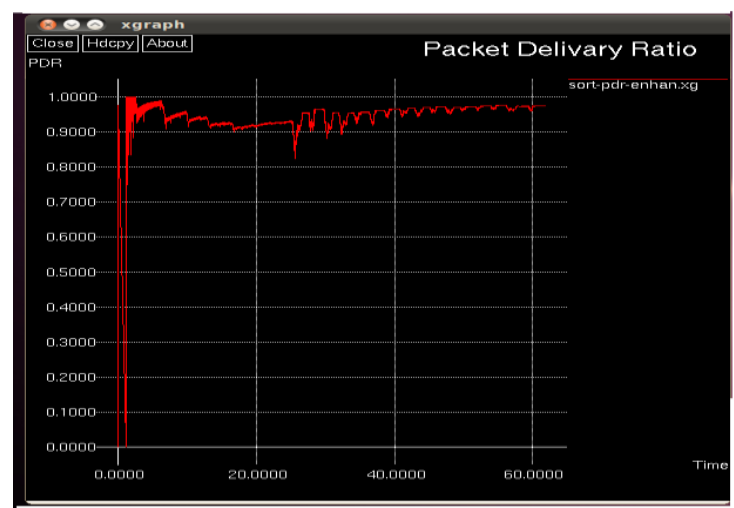

Figure 12: PDR of enhancement system

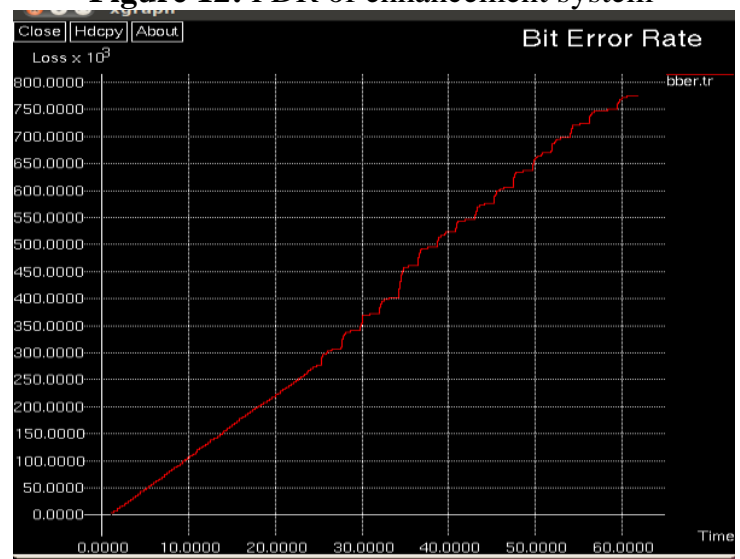

Figure 13: Bit error rate of enhancement system

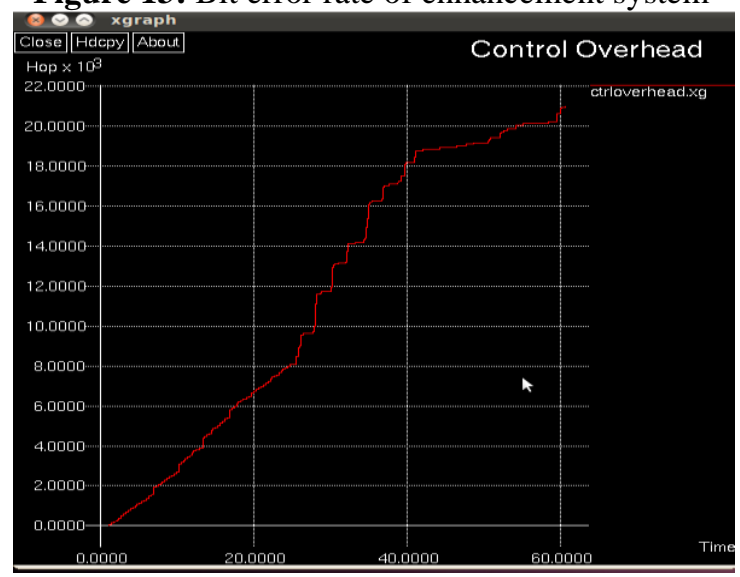

Figure14: Control overhead of enhancement system

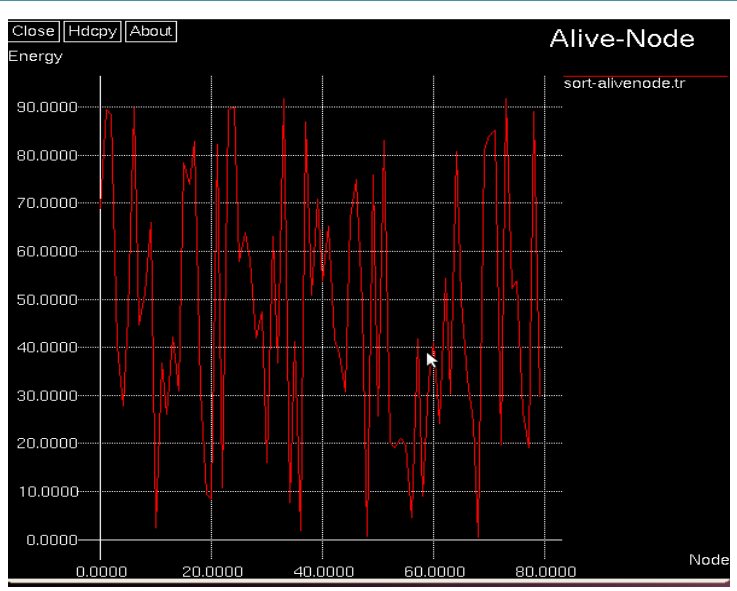

Figure15: alive nodes of enhancement system

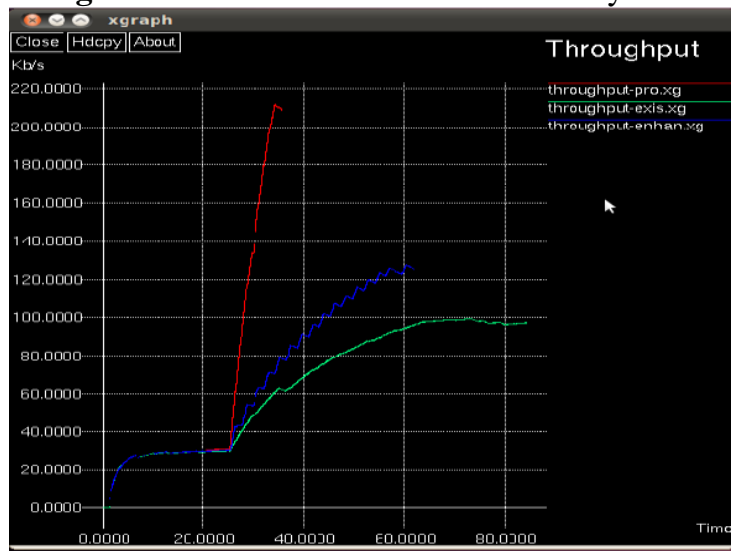

Figure16: comparisons for throughput with proposed and existing system

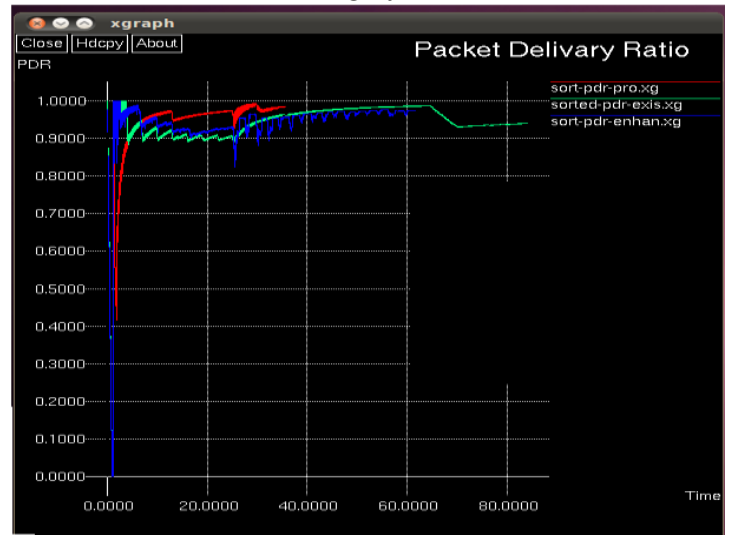

Figure17: comparisons for packet delivery ratio with proposed, enhancement and existing system

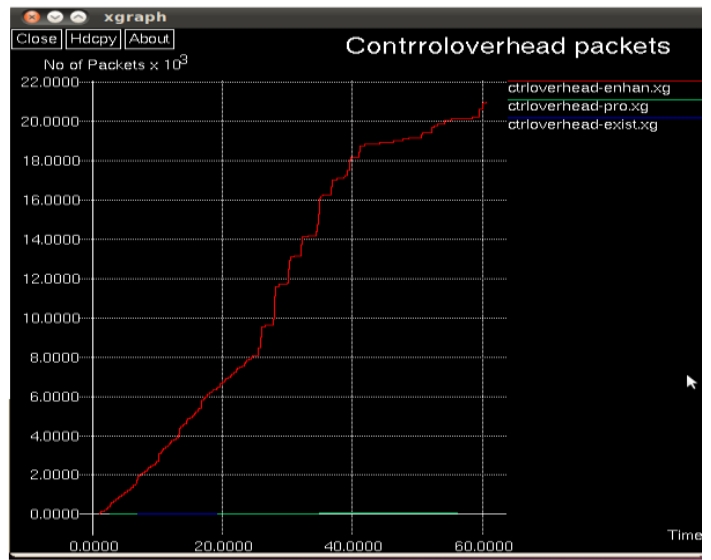

Figure18: comparisons for control over head with proposed, enhancement and existing system.

Volume 5 Issue 4, April 2016 


\section{International Journal of Science and Research (IJSR) \\ ISSN (Online): 2319-7064}

Index Copernicus Value (2013): 6.14 | Impact Factor (2015): 6.391

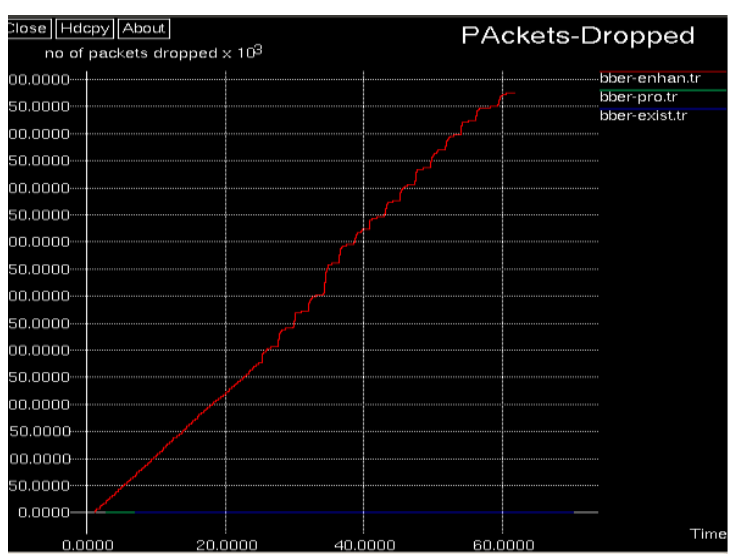

Figure19: comparisons for packets-dropped with proposed, enhancement and existing system.

\section{Simulation Summary}

Simulation result has different matrices are introduced for the network performance. These result shows, existing and proposed, enhancement system Figure 2, 3,4, $5,6,7,8,9.10,11,12,13,14,15$ and figure $16,17,18,19$ are the comparison of existing, proposed and enhancement system of the performance matrices. Data packets enabled along with source nodes and successfully reached to the base station and introduced the efficient use of clustering in SPIN-S and significant performance gains over the AODV for lifetime of network, stability and network alive.

\section{Conclusion}

In this paper attempts to provide the guarantee the data to base station along with stability and better network life time and energy used in efficiently also cluster head will be equal responsibility. Security will be taken for further studies where a normal node can be hacker so it should be result this part done will be feature work.

\section{References}

[1] I. Akyildiz, W. Su, Y. Sankarasubramaniam, and E. Cayirci, “A survey on sensor networks," IEEE Communications Magazine, vol. 40, no. 8,pp. 102-114, August 2002.

[2] Malik Tubaishat and Sanjay Madria, "Sensor Networks: An Overview," IEEE Potential, pp. 20-23, 2003.

[3] Holger Karl, and Andreas Willig, "Protocol and Architecture for Wireless Sensor Networks," Wiley Publication, 2005.

[4] D. Estrin, R. Govindan, "Next Century Challenges: Scalable Coordination in Sensor Networks," MobiCom 1999, pp. 263-270, 1999.

[5] J. Carle, and D. Simplot-Ryl, "Energy-Efficient Area Monitoring for Sensor Networks," Computer, Vol.37, pp 40-46, 2004.

[6] Po-Jen Chuang, "Efficient Data Gathering Scheme for Wireless Sensor Networks,” Springer, pp. 370-379, 2005. 